\title{
Urgencias en patología neuromuscular
}

\section{Emergencies in neunomuscular pathology}

\author{
T. Ayuso, I. Jericó
}

\section{RESUMEN}

La debilidad muscular aguda (DMA) es el síntoma predominante de las urgencias neuromusculares, especialmente si afecta a la musculatura respiratoria u orofaríngea. La DMA es un síndrome plurietiológico y con distintos niveles lesionales en la unidad motora. Dentro del amplio grupo de enfermedades neuromusculares, las que con mayor frecuencia provocan DMA e insuficiencia respiratoria son el síndrome de GuillainBarré (SGB) y la miastenia gravis (MG).

El SGB constituye la causa más frecuente de parálisis flácida aguda; puede ocasionar fallo respiratorio en un tercio de los casos precisando ventilación mecánica. El diagnóstico preciso de este síndrome permitirá iniciar tratamiento inmunomodulador, que ha demostrado que modifica el curso de la enfermedad. Además, la valoración clínica de los pacientes y el conocimiento de sencillos tests neurofisiológicos y de función respiratoria guiarán la decisión de ventilación mecánica evitando la intubación de urgencia.

La urgencia más frecuente que ocasiona la MG es la crisis miasténica, definida por el deterioro en la función bulbar con insuficiencia respiratoria aguda y riesgo de parada respiratoria. Ocurre en un $15-20 \%$ de pacientes miasténicos y puede desencadenarse por múltiples factores. Además del diagnóstico preciso de la crisis es importante la supresión de los factores desencadenantes y medidas de soporte ventilatorio. Entre las medidas farmacológicas son la plasmaféresis y las inmunoglobulinas intravenosas los instrumentos más útiles en la actualidad; estos tratamientos no sustituyen la vigilancia intensiva y el reconocimiento de los signos inminentes de fallo respiratorio que implican soporte ventilatorio invasivo o no invasivo.

Palabras clave. Debilidad muscular aguda. Síndrome de Guillain-Barré. Crisis miasténica. Insuficiencia respiratoria neuromuscular.

An. Sist. Sanit. Navar. 2008; 31 (Supl. 1): 115-126.

\begin{abstract}
Acute muscle weakness (AMW) is the predominant symptom of neuromuscular emergencies, especially if it affects the respiratory or oropharyngeal musculature . AMW is a multi-etiological syndrome, with different lesion levels in the motor unit. Within the broad group of neuromuscular diseases, those that most frequently provoke AMW and respiratory failure are Guillain-Barré syndrome (GBS) and myasthenia gravis (MG).

GBS is the most frequent cause of acute flaccid paralysis; it can cause respiratory failure in a third of cases, making mechanical ventilation necessary. Accurate diagnosis of this syndrome enables immunomodulatory treatment to be started, which has been shown to modify the course of the disease. Besides, clinical evaluation of the patients and knowledge of the simple tests of neurophysiology and respiratory function will guide the decision on mechanical ventilation, avoiding emergency intubation.

The most frequent emergency caused by MG is myasthenic crisis, defined by the deterioration in the bulbar function with acute respiratory insufficiency and risk of respiratory stoppage. This occurs in $15-20 \%$ of myasthenic patients and can be triggered by numerous factors. Besides early identification of the crisis, it is important to suppress the triggering factors and to provide measure of ventilatory support. Amongst the pharmacological measures, the most useful instruments at present are plasmapheresis and intravenous immunoglobulins; these treatments do not cancel the need for intensive vigilance and of checking for imminent signs of respiratory failure that will involve invasive or non-invasive ventilatory support.
\end{abstract}

Key words. Acute muscle weakness. Guillain-Barré síndrome. Myasthenic crisis. Neuromuscular respiratory insufficiency.
Servicio de Neurología. Hospital de Navarra. Pamplona.
Correspondencia:

Teresa Ayuso Blanco

Servicio de Neurología

Hospital de Navarra

C/ Irunlarrea, 3

31008 Pamplona

Tfno. 848422291

E-mail: tayusob@yahoo.es 


\section{INTRODUCCIÓN}

El síntoma básico en urgencias neuromusculares es la debilidad motora, expresión de patología neuromuscular preexistente o enfermedad neurológica de comienzo. Se define debilidad muscular aguda (DMA) como la pérdida de fuerza instaurada en horas o días (menos de 4 semanas) más o menos generalizada causada por alteración en algún punto de la unidad motora (UM). La UM es la unidad anatómica del sistema neuromuscular integrada por el cuerpo celular de la neurona motora del asta anterior o de los núcleos motores del tronco cerebral, su axón y todas las fibras musculares inervadas por dicho axón.

La DMA constituye una urgencia médica sobre todo si afecta a musculatura orofaríngea y respiratoria; supone el 0,26-1,1\% de ingresos en unidad de cuidados intensivos (UCI) precisando el 60\% de estos pacientes ventilación asistida.

La DMA es un síndrome plurietiológico y con diferente topografía lesional según el nivel lesional de la unidad motora. En la valoración del paciente con DMA hemos de considerar otras causas de debilidad generalizada, excluyendo patología de sistema nervioso central especialmente enfermedades de troncoencéfalo o medulares. Una historia clínica detallada y una exploración clínica precisa permitirá localizar el nivel lesional y así dirigir los estudios complementarios necesarios para confirmar la sospecha diagnóstica (Tabla 1).

\section{ENFERMEDADES DE LA NEURONA MOTORA}

\section{Poliomielitis}

Es una enfermedad producida por un enterovirus; es excepcional su presentación en países donde la vacunación está establecida. Cursa con debilidad asimétrica que evoluciona en días y se acompaña de fiebre, meningitis aséptica, arreflexia, fasciculaciones y atrofia. Cuadros clínicos similares pueden originarse por el virus de la parotiditis e infecciones por herpes.

Tabla 1. Etiología de la debilidad muscular aguda según nivel lesional.

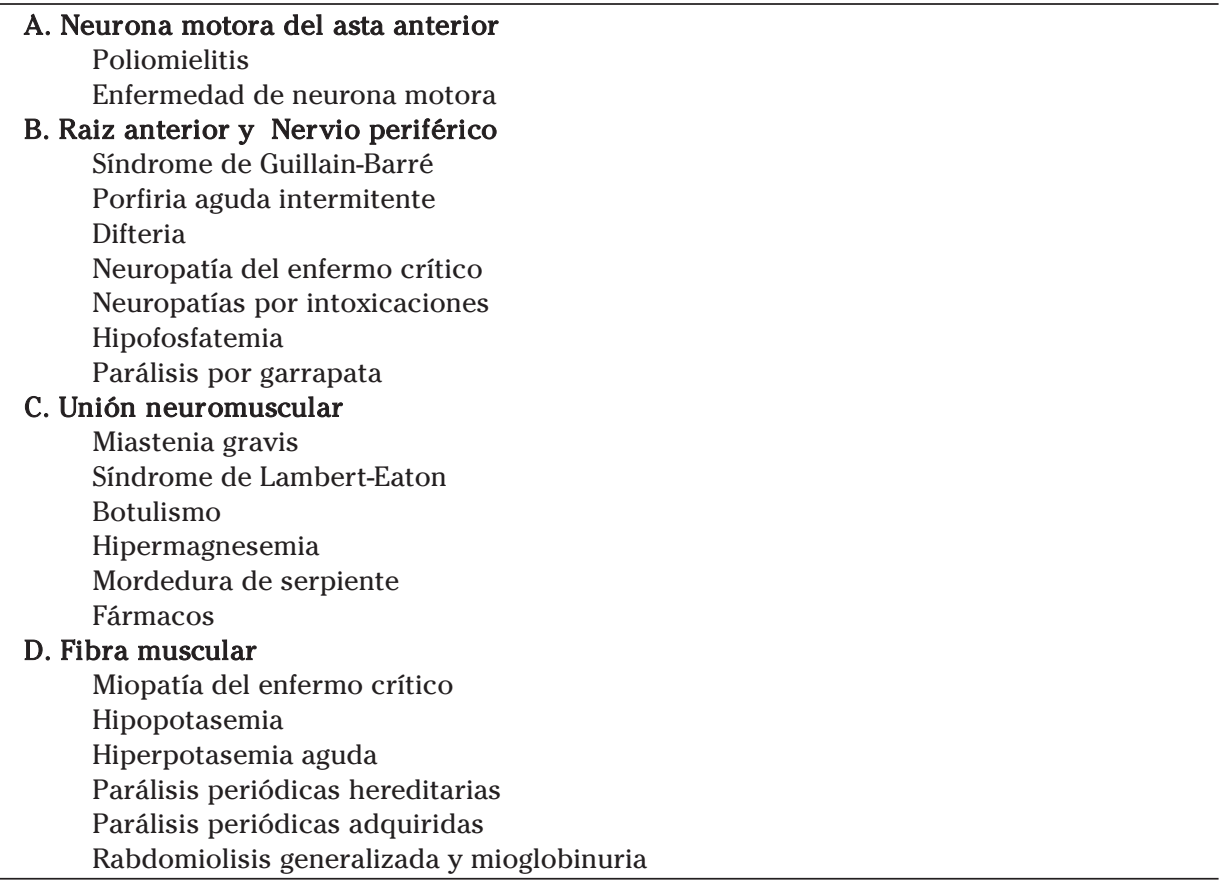


Décadas después de que la parálisis por poliomielitis se haya recuperado o sobre las secuelas residuales, en un $30 \%$ de los supervivientes, pueden reaparecer síntomas de debilidad, dolor y fatiga que afectan a la misma extremidad y constituyen el síndrome postpolio.

\section{Enfermedad de neurona motora}

La esclerosis lateral amiotrófica (ELA) se caracteriza por síntomas y signos de degeneración de neuronas motoras superiores e inferiores que lleva a debilidad progresiva de la musculatura bulbar, de extremidades, musculatura torácica y abdominal. No se presenta como DMA sino como torpeza progresiva y la aparición de insuficiencia respiratoria es insidiosa, habitualmente en fases avanzadas.

La complejidad de esta enfermedad y ausencia de tratamiento curativo hace preciso un diagnóstico temprano y seguimiento neurológico y multidisciplinar próximo que mejore la calidad de vida de estos pacientes y evite situaciones de urgencias no previstas ${ }^{1}$.

\section{ENFERMEDADES DE NERVIO PERIFÉRICO Y RAÍCES}

\section{Síndrome de Guillain Barré (SGB)}

Es una polirradiculopatía desmielinizante inflamatoria aguda de patogenia disinmune; constituye la causa más frecuente de parálisis flácida aguda que afecta a cualquier edad, sobre todo a adultos jóvenes y por encima de los 55 años con una incidencia de 1-4 por 100.000 habitantes/año en los diferentes estudios ${ }^{2,3}$. Es frecuente un pródromos de infección vírica o bacteriana; la infección respiratoria suele preceder a las formas más graves detectándose en el 18\% de los casos anticuerpos a Cytomegalovirus y otras veces infección gastrointestinal por Campylobacter jejuni. Otros gérmenes implicados en la patogénesis del SGB son Mycoplasma pneumoniae, virus de Epstein-Barr, HIV y virus de Hepatitis A.

El comienzo clínico habitual consiste en debilidad en extremidades inferiores, dolor en piernas y región lumbar y pares- tesias distales. El déficit motor es simétrico con afectación proximal y distal; puede ocasionar fallo respiratorio en el 33\% de los casos precisando ventilación mecánica. Es frecuente la afectación de pares craneales especialmente el facial. La disfunción sensitiva es de tipo irritativo con dolor y parestesias que son el síntoma inicial en el $50 \%$ de los casos, también presentan disestesias en la fase de recuperación. Los reflejos osteotendinosos (ROT) están abolidos en el 70\% de los casos aunque se conservan en fases iniciales y progresan a la supresión en 7 días.

Hay disfunción autonómica con arritmias y cambios tensionales en el $60 \%$ de los casos.

Es una enfermedad autolimitada que progresa en 2-3 semanas, presentando su máxima gravedad a los 9 días, y posterior recuperación en semanas o meses; la mortalidad oscila según las series del 2 al $11 \%^{4,5}$ llegando al $20 \%$ en los pacientes que requieren ventilación mecánica ${ }^{6}$.

Se considera el SGB como un espectro clínico que comprende un grupo heterogéneo de entidades con diferentes manifestaciones clínicas, histológicas y etiopatogénicas. Según el patrón clínico, fibras afectadas y tipo de lesión se clasifica en cuatro variantes $^{7}$ :

1. Polirradiculopatía inflamatoria desmielinizante aguda (AIDP).

2. Neuropatía aguda sensitivomotora axonal (AMSAN).

3. Neuropatía aguda motora axonal (AMAN): se asocia al antecedente de infección por Campylobacter jejuni; en muchos casos hay IgG antiGM1.

4. Síndrome de Miller-Fisher (SMF): se caracteriza por oftalmoplejia, ataxia y arreflexia, puede desencadenarse por una infección entérica y es característica la presencia de anticuerpos antigangliósido GQ1b que reconocen epítopos expresados en el nodo de nervios oculomotores.

El diagnóstico se basa en las características clínicas, y se apoya en datos del laboratorio y el estudio neurofisiológico 
(Tabla 2). El líquido cefalorraquídeo (LCR) los primeros días puede ser normal presentando a partir de la primera semana aumento de proteinas y mínima o ausente pleocitosis linfocítica (disociación albúmina-citológica). Los estudios inmunológicos séricos son también útiles para el diagnóstico confirmando la sospecha clínica; la asociación más sólida es la presencia de anticuerpo de la clase IgG anti-GQ1b en el $96 \%$ de la variante de SMF. Otros anticuerpos dirigidos contra gangliósidos y otros glicolípidos de las estructuras neurales tiene también una alta prevalencia en SGB, incluyendo anti-GM1b, GM2, GD1a, GalNAc-GD1a, GD1b, GT1a, GT1b y GM18.

El estudio electrofisiológico puede ser normal o inespecífico en el $20 \%$ hasta la segunda o tercera semana; después lo más frecuente es la presencia de una polineuropatía multifocal desmielinizante, en el $80 \%$ de los casos se encuentra disminución de velocidades de conducción (VC) o bloqueos de la conducción en algún momento. La
$\mathrm{VC}$ es $<60 \%$ de lo normal, pero la afectación es a parches y no todos los nervios se afectan. Las latencias pueden aumentar hasta el triple de su valor normal y las ondas $\mathrm{F}$ se encuentran ausentes o con latencias alargadas.

El tratamiento sintomático incluye las medidas de soporte respiratorio ${ }^{9}$, prevención de tromboembolismo pulmonar, monitorización y tratamiento de la disautonomía y de las infecciones.

El tratamiento específico o inmunomodulador que ha demostrado que altera favorablemente el curso natural del SGB son inmunoglobulinas intravenosas (IgIv) y la plasmaféresis. Está indicado dicho tratamiento en pacientes con incapacidad para caminar y en las 2 primeras semanas de evolución; probablemente sea eficaz también en fases iniciales y en debilidad moderada y en las variantes como SMF con un curso natural más benigno ${ }^{10}$. Los corticoides no han demostrado eficacia ${ }^{11}$.

Tabla 2. Criterios diagnósticos del síndrome de Guillain-Barré.

1. Datos que se requieren para el diagnóstico

-Debilidad motora progresiva en más de una extremidad

-Arreflexia

2. Datos que apoyan el diagnóstico

-Progresión inferior a 4 semanas

-Relativa simetría

-Síntomas o signos sensitivos mínimos

-Afectación de pares craneales (especialmente facial bilateral)

-Inicio de la recuperación 2-3 semanas tras progresión

-Afectación autonómica

-No fiebre al inicio

-Líquido cefalorraquídeo: elevación de proteínas a partir de la primera semana y menos

de 10 células

-Estudio electrofisiológico típico

3. Datos que hacen dudar del diagnóstico

-Asimetría marcada y persistente

-Alteración esfinteriana persistente

-Alteración esfinteriana vesical o rectal inicial

-Pleocitosis superior a 50 células mononucleares $/ \mathrm{mm}$

-Presencia de polimorfonucleares en líquido cefalorraquídeo

-Nivel sensitivo claro

4. Datos que descartan el diagnóstico

-Neuropatía tóxica por hexacarbonos (n-hexano), plomo, organofosforados o fármacos

-Diagnóstico definitivo de poliomielitis, botulismo, porfiria, difteria

-Síndrome puramente sensitivo 
Las Iglv a dosis de $2 \mathrm{gr} / \mathrm{kg}$ de peso repartidas en 2-5 días, mejoran la enfermedad y son la terapia primaria por ser tan eficaces como la plasmaféresis ${ }^{12,13}$, su inferior coste, menores efectos secundarios, fácil administración y posibilidad de utilizarse en niños y hospitales comarcales. Se ha probado la combinación de IgIv con metilprednisolona sin demostrar mayor eficacia que con IgIv solas; están en marcha otros ensayos para valorar el efecto adicional de inmunosupresores como mofetil micofenolato asociados a IgIv ${ }^{14}$.

La plasmaféresis también ha demostrado mejoría en estudios controlados ${ }^{15}$; deben efectuarse 4-5 recambios de 2 a $4 \mathrm{~L}$ cada uno en 7-10 días. Es el tratamiento de elección cuando hay efectos secundarios con IgIv, insuficiencia cardiaca congestiva, insuficiencia renal y déficit de IgA.

\section{OTRAS ENFERMEDADES DE NERVIO PERIFÉRICO}

\section{Porfiria aguda intermitente}

Es una enfermedad hereditaria autosómica dominante más frecuente en mujeres. Se debe a la conjunción de un déficit de la actividad de la enzima porfobilinógeno(PBG)-desaminasa (también llamada uroporfirinógeno I sintetasa) y determinados factores desencadenantes. Las crisis típicas se inician con dolor abdominal y en $75 \%$ de los casos hay clínica neurológica siendo lo más habitual la neuropatía tipo SGB axonal.

La polirradiculopatía puede afectar a músculatura respiratoria y requerir ventilación mecánica. Son frecuentes (70\%) las manifestaciones neuropsiquiátricas y puede haber crisis convulsivas hasta en el $20 \%$. El diagnóstico se basa en la detección de elevación de ácido delta-amino-levulínico (ALA), PBG y uroporfirina en orina durante los ataques. Es esencial comprobar déficit de PBG-desaminasa para estudio de portadores. El estudio de LCR es normal.

El estudio neurofisiológico es de polineuropatía axonal mixta de predominio motor. El tratamiento se basa en la prevención, evitando factores desencadenantes, e infusión de hematina para inhibir síntesis de ALA sintetasa durante los ataques.

\section{Difteria}

Es una enfermedad excepcional en nuestro entorno por la vacunación. La clínica debuta con una infección faríngea seguida de polineuropatía sensitivo-motora desmielinizante, borrosidad visual y afectación bulbar precoz.

La polineuropatía se instaura a partir de las 4-8 semanas y puede simular un $\mathrm{SGB}$, la borrosidad visual y la afectación bulbar precoz deben hacen pensar en difteria. El tratamiento con antitoxina diftérica en las primeras $48 \mathrm{~h}$ disminuye la frecuencia y gravedad de las complicaciones, no siendo eficaz una vez iniciada la polineuropatía.

\section{Neuropatía del enfermo crítico}

Ocurre en pacientes con estancias prolongadas en UCI, especialmente con sepsis o fallo multiorgánico. Las principales manifestaciones clínicas son la dificultad para extubar y debilidad de predominio en extremidades inferiores o tetraplejia. No hay afectación de pares craneales, los ROT están disminuidos o abolidos. Hay atrofia muscular y la alteración sensitiva es constante. En la fisiopatogenia se han implicado múltiples factores: déficits nutricionales, uso de aminoglucósidos, bloqueantes neuromusculares, alteraciones hidroelectrolíticas y fallo multiorgánico.

En el estudio neurofisiológico hay signos de disfunción axonal de predominio motor con latencias distales y estimulación repetitiva normales. Con frecuencia hay datos de miopatía asociada siendo preferible para algunos autores utilizar el término de mioneuropatía del enfermo crítico ${ }^{16}$.

El diagnóstico diferencial se plantea con causas neurológicas centrales, bloqueo neuromuscular prolongado, miopatía del enfermo crítico o enfermedad neurológica preexistente como SGB. La mortalidad media es del $35 \%$ aunque si hay fallo multiorgánico oscila del 50 al $60 \%$. Puede haber recuperación completa en los casos que sobreviven al periodo crítico aunque precisan tratamiento fisioterápico intensi- 
vo y en ocasiones hay secuelas incapacitantes ${ }^{16}$.

El tratamiento es sobre todo preventivo, el control intensivo de la hiperglucemia con insulina y las medidas del tratamiento del shock séptico evitan mayor daño neuropático.

\section{Neuropatías por intoxicaciones}

Múltiples tóxicos, fármacos y déficits nutricionales pueden provocar una polineuropatía aunque generalmente son de curso subagudo o crónico y rara vez debutan con DMA. Citamos aquí las que con más frecuencia pueden ser motivo de consulta urgente.

Arsénico. Se encuentra en rodendicidas, insecticidas y pesticidas siendo la intoxicación accidental, homicida o suicida. Si la ingesta de arsénico es abundante, se caracteriza por cuadro gastrointestinal seguido de encefalopatía con disminución de conciencia y muerte en $24 \mathrm{~h}$; si se sobrevive al episodio agudo clínica de polineuropatía axonal sensitivo-motora entre los días 10 y 20 que puede progresar hasta la $5^{\text {a }}$ semana. Las formas severas pueden originar debilidad progresiva afectando a tronco y requiriendo ventilación mecánica.

Hexacarbonos. Rara vez originan DMA, con mayor frecuencia neuropatía subaguda-crónica sensitivo-motora. La intoxicación ocurre por exposición laboral o adicción a colas o disolventes. En estos últimos la instauración es más rápida, con síntomas autonómicos, debilidad generalizada y oftalmoplejia que plantea diagnóstico diferencial con SGB.

Marisco. Toxinas marinas como la saxitoxina ocasionan parálisis y depresión respiratoria.

Fármacos. Vincristina, litio, talio, disulfiram, amiodarona...ocasionan polineuropatía sensitivo-motora, generalmente axonal de curso subagudo y que obliga a la supresión del fármaco.

Hipofosfatemia. Desencadenada en nutrición parenteral sin fósforo puede producir confusión, somnolencia y coma y parálisis aguda similar al SGB.

Parálisis por garrapata. Es una parálisis flácida ascendente similar a SGB, sin alteraciones sensitivas y LCR normal. Está causada por toxina inoculada por gran número de especies de garrapatas.

\section{ENFERMEDADES DE LA UNIÓN NEUROMUSCULAR}

\section{Miastenia gravis (MG)}

La MG adquirida es una enfermedad autoinmune producida por anticuerpos que bloquean el receptor postsináptico de la acetilcolina en la unión neuromuscular. Es frecuente su asociación con otras enfermades autoinmunes y con patología del timo en el $70 \%$ de los casos (timoma en 10 $15 \%$ y en el resto hiperplasia tímica).

Las características clínicas primordiales de la debilidad muscular en la MG son la fatigabilidad, fluctuación a lo largo del día, especial localización, reproductibilidad mediante maniobras dirigidas a demostrar fatigabilidad y mejoría con anticolinesterásicos. Los músculos más frecuentemente afectados en MG son el elevador de los párpados, la musculatura ocular extrínseca, la musculatura facial y masticatoria, lengua, músculos faríngeos, laríngeos, flexores cervicales, respiratorios, músculos del tronco, del abdomen, musculatura proximal y distal en extremidades inferiores y proximal en extremidades superiores.

Se distinguen básicamente dos formas clínicas: la miastenia ocular y la generalizada. La MG ocular es aquella en la que las manifestaciones clínicas se restringen a la musculatura ocular extrínseca aunque por encima del $80 \%$ se generalizarán en los 2 años posteriores. Es la forma clínica más benigna aunque genera dudas diagnósticas y terapeúticas ${ }^{17}$. La forma generalizada de la enfermedad se produce cuando están implicados músculos extraoculares, haya o no también participación ocular. Cuando los síntomas afectan de manera predominante a extremidades se consideran síntomas A y si predominan en la musculatura bulbar se consideran síntomas $\mathrm{B}$.

El diagnóstico se basa en cuatro puntos: 1. clínica sugestiva y exploración que ponga de manifiesto la fatiga muscular, 2. Test farmacológico positivo que consiste 
en mejoría sintomática tras inyección de Edrofonio, 3. estudio electrofisiológico dirigido a demostrar el trastorno en la transmisión neuromuscular mediante la estimulación repetitiva y electromiograma de fibra única que valora el jitter o tiempo diferencial entre la activación de dos fibras musculares de la misma unidad motora; el aumento del jitter ocurre en $88-99 \%$ de pacientes con MG, 4. determinación de anticuerpos anti-receptor de acetil-colina. Pueden estar ausentes o a bajos títulos en el 50\% de la MG ocular o infantil. En las formas seronegativas pueden detectarse IgG anti-MuSK, anticuerpos dirigidos a una proteína intrínseca de membrana, tirosina quinasa músculo-específica, que pueden llegar a detectarse, según las series en el 34-71\% de las MG generalizada seronegativas y configuran un subgrupo clínico, evolutivo y de respuesta terapéutica diferente a aquellas en las que el antígeno es el receptor de la acetil-colina ${ }^{18,19}$.

Existen dos tipos de tratamiento ${ }^{20}$, el sintomático dirigido a prolongar la acción de la acetil-colina en la membrana postsináptica y mejorar así la transmisión neuromuscular y el etiopatogénico o modificador de la historia natural de la enfermedad que engloba la timectomía, los agentes inmunosupresores e inmunomoduladores a corto plazo como la plasmaféresis o las IgIv.

Loa anticolinesterásicos actúan en la sinapsis colinérgica inhibiendo la acetilcolinesterasa; el más usado es el bromuro de piridostigmina cuya acción comienza a los 15-30 minutos y dura de 3 a 4 horas; la dosis debe ajustarse según la respuesta clínica y la aparición o no de efectos adversos. La timectomía está indicada en la MG generalizada en pacientes comprendidos entre los 8-55 años y si hay timoma por el riesgo de extensión del tumor a estructuras mediastínicas; la eficacia ocurre a partir de los 6 meses de la cirugía y es mayor cuando la enfermedad comienza antes de los 50 años y si se realiza durante los 2 primeros años de la enfermedad en caso de hiperplasia tímica.

Dentro de los tratamientos dirigidos a modificar la respuesta inmune los corticoides siguen siendo el principal pilar en la MG; son eficaces para inducir remisión de la enfermedad en el 50-80\% de los casos e inducir mejoría en todos los grados de debilidad. Dosis altas iniciales pueden empeorar los síntomas por lo que se recomienda iniciar la medicación con dosis de $15-20 \mathrm{mg} /$ día e incremento gradual o dosis de $1 \mathrm{mg} / \mathrm{kg} /$ día si se realiza en régimen de ingreso hospitalario y monitorizando función respiratoria.

La urgencia más frecuente que acontece en esta entidad es la crisis miasténica (CM), definida por el deterioro en la función bulbar con insuficiencia respiratoria aguda y riesgo de parada respiratoria. Ocurren en un $15-20 \%$ de pacientes miasténicos en algún momento de la enfermedad y pueden desencadenarse por infecciones, enfermedades sistémicas, sobrecarga emocional o embarazo; la mortalidad es del $4 \%$.

El tratamiento de las $\mathrm{CM}$ ha de ir dirigido a mejorar de la forma más rápida posible la transmisión neuromuscular. Además de la identificación precoz de la crisis es importante la supresión de los factores desencadenantes y medidas de soporte ventilatorio.

Dentro de las medidas farmacológicas son la plasmaféresis y las IgIv los instrumentos más útiles en la actualidad. La utilización de inhibidores de la acetil-colinesterasa en $\mathrm{CM}$ ha disminuido en las últimas décadas por su variable vida media y por poder dificultar la la extubación generando confusión entre crisis colinérgica y $\mathrm{CM}$; por otra parte el aumento de dosis de corticoides no supone un beneficio en la fase aguda sino, como ya hemos comentado, empeorar inicialmente la debilidad muscular.

La plasmaféresis elimina rápidamente anticuerpos patogénicos y otros componentes plasmáticos como moléculas de adhesión y citoquinas. El tratamiento estándar es de 5-6 sesiones a días alternos. Las nuevas técnicas aferéticas como plasmaféresis con doble filtración e immunoadsorción tienen efectos terapéuticos y adversos similares ${ }^{21}$.

Altas dosis de inmunoglobulinas por via iv ( $2 \mathrm{~g} / \mathrm{kg}$ de peso) han demostrado eficacia en CM y en dos ensayos randomizados doble ciego $^{22,23}$ no se encuentran diferencias significativas respecto a la plasmaféresis aunque otros autore ${ }^{21}$ encuentran 
una recuperación más rápida y predecible con plasmaféresis. Además, en crisis miasténicas hay consenso clínico y un ensayo randomizado sobre la utilidad de inmunoglobulinas en formas clínicas con gravedad moderada ${ }^{24}$.

\section{Botulismo}

Está causado por la exotoxina del Clostridium botulinum que bloquea la liberación presináptica en la unión neuromuscular; hay 7 tipos de toxinas, de ellas la A es la más grave requiriendo en el $67 \%$ de los pacientes ventilación mecánica. La clínica se inicia de 12 a $36 \mathrm{~h}$ tras ingesta de comida contaminada (margen de 2 horas a 1semana) con sintomatología bulbar (disfagia, disartria, parálisis oculomotora) y disfunción autonómica con midriasis arreactiva, boca seca, estreñimiento o retención urinaria, expresión del bloqueo parasimpático. El grado de debilidad muscular es variable pudiendo presentar parálisis flácida con progresión descendente proximal y en ocasiones afectación respiratoria. La ausencia de síntomas sensitivos es útil en el diagnóstico diferencial con SGB aunque hay dudas diagnósticas con el SMF. El examen del LCR es normal. Salvo complicaciones, la recuperación es total aunque pueden permanecer como secuelas fatiga o sequedad de boca.

El diagnóstico es de sospecha ante el cuadro clínico y los datos epidemiológicos: ingesta de conservas caseras mal esterilizadas. Se confirma con la detección de toxina en suero, heces o restos de comida contaminada y test de toxicidad en ratón y se apoya con el estudio neurofisiológico que cursa con reducción de potencial motor evocado, un $20 \%$ de incremento en estimulación repetitiva, potenciales miopáticos en EMG y aumento marcado del jitter.

El tratamiento más importante es el mantenimiento de un soporte vital adecuado con vigilancia en UCI en casos graves y ventilación mecánica si es necesaria. La utilización de antibióticos no es eficaz, únicamente en forma infantil y formas ocultas del adulto y la antitoxina sólo si su administración es muy precoz. El uso de guanidina y aminopiridina es de escaso beneficio.

\section{Síndrome de Lambert -Eaton}

Es un síndrome miasteniforme caracterizado por debilidad proximal con fatiga que mejora con ejercicio breve; se presenta de forma aguda, subaguda o crónica y la debilidad predomina en musculatura de cintura pélvica. Se asocia a disminución o ausencia de ROT y síntomas disautonómicos. Se debe a un trastorno en la trasmisión neuromuscular autoinmune por presencia de anticuerpos dirigidos a canales de calcio en membrana presináptica. La mitad de los casos tienen una neoplasia oculta, que en el $80 \%$ de ellos es un cáncer pulmonar de células pequeñas.

A diferencia de MG, la afectación ocular es menos frecuente siendo el comienzo de la enfermedad solo en el $5 \%{ }^{25}$, la debilidad predomina en piernas y no suele ocasionar insuficiencia respiratoria.

El estudio neurofisiológico confirma el trastorno presináptico mostrando incremento de la amplitud del potencial evocado motor en la estimulación repetitiva de altas frecuencias.

El tratamiento sintomático con diaminopiridina y piridostigmina mejora la fatiga y debilidad muscular aunque la respuesta es muy variable. Es fundamental el tratamiento del cáncer si lo hubiera y en la formas idiopáticas, inmunosupresión con prednisona, plasmaféresis o Iglv.

\section{MIOPATÍAS}

\section{Miopatía del enfermo crítico}

La debilidad muscular primaria en pacientes de UCI puede ser el trastorno neuromuscular adquirido más común de pacientes críticos; clínicamente cursa con debilidad generalizada en extremidades, atrofia, hiporreflexia y dificultad para la extubación siendo con frecuencia difícil de distinguir de la polineuropatía del enfermo crítico $^{16}$.

Se da preferentemente en ingresados en UCI por estatus asmático; la sepsis y sus mediadores de la respuesta inflamatoria juegan un importante papel, y también la denervación si hay polineuropatía asociada y fármacos, sobre todo bloqueantes neuromusculares y altas dosis de esteroi- 
des. En fases tempranas se produce una elevación de la creatin quinasa (CK).

Se han descrito 2 tipos de patrones histológicos, el de miopatía necrotizante con grados variables de necrosis de fibras y otro en el que predomina la pérdida de filamentos de miosina ${ }^{26}$, la elevación de CK es más prominente en el primer tipo y el pronóstico es peor que en el segundo.

El diagnóstico se basa en la sospecha clínica por los factores predisponentes, elevación de CK y estudios neurofisiológico e histológico.

\section{Parálisis periódicas hereditarias}

Son canalopatías genéticas que pueden presentarse como crisis de DMA y se asocian a niveles alterados de potasio. Hay 2 formas clínicas, la parálisis periódica hipopotasémica primaria (PPhK) y parálisis hiperpotasémica primaria $(\mathrm{PPHK})^{7}$. La PPhK es una enfermedad autosómica dominante causada por mutaciones en el gen que codifica una subunidad del canal del calcio del músculo esquelético. Se inicia de los 10 a 30 años; frecuentemente se presentan con crisis agudas de debilidad flácida en las 4 extremidades y arreflexia, sin afectación bulbar. Son factores desencadenantes el ejercicio, el frío y la ingesta de hidratos de carbono. El diagnóstico se realiza por datos clínicos, historia familiar y determinación de potasio sérico disminuido (2-3 $\mathrm{mEq} / \mathrm{l}$ ) durante los episodios. El tratamiento consiste en grandes dosis de potasio oral o solución iv de cloruro potásico y la prevención con dieta rica en potasio, baja en hidratos de carbono e hiposódica, espirolactona, y acetazolamida.

La parálisis periódica hiperpotasémica es autosómica dominante por mutaciones en un gen que codifica una subunidad del canal de sodio de las fibras musculares. Comienza en la infancia o segunda década de vida y cursa con crisis de gravedad variable tras ejercicio, frío y ayuno que suelen durar de 1 a 2 horas. El nivel de potasio sérico es alto o en el límite. Si la crisis es aguda puede tratarse con $2 \mathrm{~g} / \mathrm{kg}$ de glucosa vía oral e insulina subcutánea o con gluconato cálcico iv al $10 \%$.

\section{Parálisis periódicas secundarias}

\section{Hipopotasemia}

Produce debilidad en el curso de horas o días en pacientes con déficit crónico de potasio ( $<$ a $3 \mathrm{mEq} / \mathrm{L}$ ) que sufren una pérdida adicional. Los casos leves sólo cursan con debilidad en extremidades inferiores de predominio proximal a diferencia de las forma graves en las que hay progresión a brazos y músculos cervicales y respiratorios. El tratamiento consiste en administrar cloruro potásico.

\section{Hiperpotasemia}

Suele ocurrir en contexto de una insuficiencia renal o suprarrenal crónica en las que se produce aporte adicional de potasio. Produce una debilidad flácida de inicio en piernas que se generaliza en horas o días y se acompaña de arreflexia, pudiendo llegar a producir insuficiencia respiratoria. Aparece con niveles de potasio de entre 7,5-11,5 mEq/L. El tratamiento va dirigido a evitar las arritmias con gluconato cálcico, seguido de suero glucosado e insulina, bicarbonato sódico e incluso diálisis.

\section{Rabdomiolisis}

Es definida por la lesión de las fibras musculares con liberación de la mioglobina y otros compuestos intracitoplasmáticos. La mioglobina es un pigmento de gran peso molecular que participa en el trasporte de oxígeno a través de membranas y pasa a plasma y posteriormente a orina produciendo su pigmentación (mioglobinuria $)^{7}$. Puede ocurrir en múltiples situaciones: necrosis muscular por traumatismos, inmovilización prolongada, infecciones, fármacos o tóxicos; es frecuente en miopatías inflamatorias y trastornos musculares metabólicos o hereditarios. Los síntomas predominantes son mialgias, debilidad muscular proximal o generalizada y marcada elevación de CK sérica y mioglobinuria.

El tratamiento es el la enfermedad de base y de la insuficiencia renal y las complicaciones sistémicas si las hubiera. 


\section{INSUFICIENCIA RESPIRATORIA EN PATOLOGÍA NEUROMUSCULAR}

La insuficiencia respiratoria (IR) es la complicación más temida de la patología neuromuscular. Enfermedades neuromusculares crónicas como la esclerosis lateral amiotrófica y distrofias musculares producen dificultades respiratorias generalmente en fases avanzadas originadas por la debilidad muscular o bulbar y agravadas por aspiración o infecciones respiratorias. Las decisiones para prolongar la vida mediante medidas de soporte ventilatorio se deben tomar mucho antes de que el paciente desarrolle dificultad respiratoria aguda después de una información detallada al pacientes de las ventajas e inconvenientes de estos tratamientos ${ }^{1}$.

La patología neuromuscular aguda que en nuestro medio produce con mayor frecuencia IR son el SGB y MG. Hay cuatro componentes que pueden contribuir al fracaso respiratorio. Primero, a nivel de la vía aérea superior, la debilidad facial, orofaríngea o laríngea interfiere con la deglución y el aclaramiento de secreciones generando riesgo de broncoaspiración y obstrucción mecánica al paso del aire sobre todo en decúbito. En segundo lugar, la debilidad de los músculos inspiratorios (diafragma, intercostales y musculatura accesoria) origina una inadecuada expansión pulmonar con microatelectasias que lleva a desacoplamiento ventilación/perfusión e hipoxemia. En tercer lugar, la debilidad de la musculatura espiratoria dificulta la tos adecuada y la eliminación de secreciones con el consiguiente riesgo de aspiración y neumonía. Por último, las complicaciones asociadas como neumonía o embolismo pulmonar también contribuyen al fracaso respiratorio $^{27}$.

La valoración clínica de los pacientes y los tests objetivos de función respiratoria guiarán la decisión de ventilación mecánica. Los tests de función pulmonar más comunes son la espirometría, las curvas flujo-volumen, la evaluación de músculos respiratorios mediante medición de presiones estáticas máximas inspiratorias (PImax) y espiratorias (PEmax) en boca o presión nasal tras esnifado (SNIP) y la gasometría arterial. El parámetro más importante de la espirometría es la capacidad vital (CV); frecuentemente se sustituye por el peak-flow, pero lo ideal es disponer de un espirómetro electrónico portátil para determinar CV, volumen corriente y frecuencia en la cabecera del paciente. Una caída en la CV $<10 \%$ en decúbito comparada con bipedestación refleja debilidad diafragmática. La presencia de IR en la gasometría suele ser tardía, una vez que la disfunción de la musculatura respiratoria es importante por lo que es poco sensible para detectar el deterioro de la musculatura respiratoria.

Son criterios absolutos de intubación en fracaso respiratorio agudo la pérdida de conciencia, parada respiratoria o cardíaca, disfunción bulbar con aspiración y presencia de shock, arritmias o alteraciones gasométricas. Sin embargo, no se recomienda tener que intubar con urgencia a estos pacientes, sino que la decisión debe tomarse más precozmente basándose en los signos clínicos de fatiga muscular y en las medidas de función respiratoria ${ }^{27}$.

Los signos generales de fracaso respiratorio inminente son la rápida progresión de la debilidad generalizada, disfagia, disfonía y disnea en ejercicio y en reposo. En la valoración subjetiva predicen la necesidad de monitorización la presencia de respiración rápida y superficial, taquicardia, tos débil, lenguaje entrecortado, utilización de musculatura accesoria, movimientos abdominales paradójicos, ortopnea, tos tras deglución y debilidad de trapecios y músculos cervicales. Las variables objetivas son una CV menor a $15 \mathrm{~mL} / \mathrm{kg}$ o inferior a $1 \mathrm{~L}$ o caída del $50 \%$, presión inspiratoria máxima mayor de $-30 \mathrm{~cm} \mathrm{H}_{2} \mathrm{O}$ o presión espiratoria máxima menor de $40 \mathrm{~cm}$ $\mathrm{H}_{2} \mathrm{O}$ y desaturación nocturna.

En el SGB, una de las principales causas de IR es la debilidad del diafragma; el estudio de velocidad de conducción del nervio frénico y registro del potencial de acción diafragmático realizado en los primeros días del inicio clínico pueden ser útiles para predecir fallo respiratorio $\mathrm{y}$ necesidad de intubación ${ }^{28}$.

Lawn y $\operatorname{col}^{29}$, en la revisión de 114 pacientes consecutivos con SGB grave ingresados en UCI de los que 60 precisaron 
ventilación mecánica, analizan los marcadores de riesgo de fallo respiratorio; las variables estadísticamente significativas que predecían la necesidad de VM fueron la progresión rápida de la enfermedad, la disfunción bulbar, la parálisis facial bilateral, la presencia de disautonomía y la CV inferior a $20 \mathrm{~mL} / \mathrm{kg}$, PImáx superior a -30 , PEmax menor de $40 \mathrm{~cm} \mathrm{H}_{2} \mathrm{O}$ o reducción de $>30 \%$ en CV, PImáx o PEmáx.

Predecir el curso de pacientes con SGB grave usando información clínica, estudios neurofisiológicos y tests sencillos de función respiratoria es útil para derivar a UCI y preparar intubación precoz programada. Además, los predictores de la necesidad de VM en SGB pueden generalizarse a otras enfermedades neuromusculares, si bien en MG, dada la fluctuación de la clínica, pueden ser más difíciles de aplicar.

La CM es la complicación más grave de la MG y es definida por la debilidad muscular que precisa ventilación mecánica para mantener correcta función respiratoria. Además de los desencadenantes citados (infecciones, estrés, cirugía...) son factores de riesgo de crisis asociados a la enfermedad la existencia de timoma, la presencia de debilidad muscular bulbar, antecedente de CM previa, miastenia con anticuerpos anti-MuSK y estar en fase inicial de la MG (50\% de las crisis ocurren en los 2 primeros años $)^{30}$. Los signos inminentes de fallo respiratorio son: debilidad bulbar grave; (CV <20-25 ml/kg); tos débil o respiración paradójica, estos signos predicen la necesidad de ingreso en UCI. Descenso de la CV a $15 \mathrm{~mL}$, PI máx <20-25 cm $\mathrm{H}_{2} \mathrm{O}$ y/o PE máx $<40 \mathrm{~cm} \mathrm{H}_{2} \mathrm{O}$ implican soporte ventilatorio. La hipoxemia y la hipercapnia sólo son indicadores relativos.

La ventilación no invasiva con BiPAP puede, en casos seleccionados, prevenir la necesidad de intubación ${ }^{31}$. La hipercapnia superior a $50 \mathrm{~mm} \mathrm{Hg}$ al comienzo es un predictor del fracaso de la BIPAP y necesidad de VM invasiva.

\section{BIBLIOGRAFÍA}

1. Andersen PM, Borasio GD, Dengler R, Hardiman O, Kollewe K, Leigh PN et al. Good practice in the management of amyotrophic lateral sclerosis: Clinical guidelines. An evidence-based review with good practice points. EALSC Working Group. Amyotroph Lateral Scler 2007; 8: 195-213.

2. Hughes RAC, ReEs JH. Clinical and epidemiological features of Guillain-Barré syndrome. J Infect Dis 1997; 176: S92-S98.

3. Prevots DR, Sutter RW. Assessment of Guillain-Barré syndrome mortality and morbidity in the United Status: implications for acute flaccid paralysis surveillance. J Infect Dis 1997 (suppl 1); 175: S151-S155.

4. Italian Guillain-Barré Group. The prognosis and main prognostic indicators of GuillainBarré síndrome. A multicentre prospective study of 297 patients. Brain 1996; 119: 20532061.

5. LAWN ND, WuJdICKS EF. Fatal Guillain-Barré. Neurology 1999; 52: 635-638.

6. Fletcher DD, LAWN ND, WolterTd, WiJdicks EF. Long-term outcome in patients with Guillain-Barré syndrome requiring mechanical ventilation. Neurology 2000; 54: 2311-2315.

7. Rojas-García R, Díaz-Manera, Illa I. Enfermedades del músculo, unión neuromuscular y nervio periférico. En Molina JA, Luquin MR, Jiménez-Jiménez FJ eds. Manual de diagnóstico y terapéutica neurológicos. Viguera Editores. Barcelona 2007: 485-53.

8. Kiefer R, Kieseier BC, Hartung H-P. Immune mediated neuropathies. In Pourmand $R$ and Harati Y eds. Advances in Neurology. Neuromuscular Disorders. Philadelphia: Lippincott Williams \& Wilkins 2002; 111-131.

9. WiJdicks E F M, Henderson R D, McClelLand R L. Emergency intubation for respiratory failure in Guillain-Barré syndrome. Arch Neurol 2003; 60: 947-948.

10. Mori M, Kuwabara S, Fukutake T, Hattori T. Intravenous immunoglobulin therapy for Miller Fisher síndrome. Neurology 2007; 68: 1144-1146.

11. Hughes RA, VAN DER MECHE FG. Corticosteroids for treating Guillain-Barré síndrome. Cochrane Data-base Syst Rev 2000; CD001446.

12. VAN DER Meche FG, Schmitz PI for the Dutch Guillain-Barré Group. A randomized trial comparing intravenous immune globulin and plasma Exchange in Guillain-Barré. N Engl J Med 1992; 326: 1123-1129.

13. Hughes RA, Raphael JC, Swan AV, Doorn PA. Intravenous immunoglobulin for Guillain- 
Barre syndrome. Cochrane Data-base Syst Rev 2004; (1):CD002063.

14. Garssen MPJ, van Koningsvel R, van Doorn PA. Treatment of Guillain Barré syndrome with mycophenolate mofetil: a pilot study. J Neurol Neurosurg Psychiatry 2007; 78: 1012-1013.

15. French Cooperative Group on Plasma Exchange in Guillain-Barre. Plasma Exchange in Guillain-Barre syndrome: oneyear follow-up. Ann Neurol 1992; 32: 94-97.

16. VISER LH. Critical illness polyneuropathy and myopathy: clinical features, risk factors and prognosis. Eur J Neurol 2006, 13: 1203-1212.

17. LUCHANOK U, KAMINSKI HJ. Ocular myasthenia: diagnostic and treatment recommendations and the evidence base. Curr Opin Neurol 2008, 21: 8-15.

18. Deymeer F, Gungor-Tuncer O, Yilmaz V, Parman Y, Serdaroglu P, Ozdemir C, Vincent A, SARUhAn-DiresKeneli G. Clinical comparison of anti-MuSK- vs anti-AChRpositive and seronegative myasthenia gravis. Neurology 2007; 68; 609-611.

19. Shibata-Hamaguchi A, Samuraki M, Furui E, Iwasa K, YoshiKawa H, Hayashi S, Yamada M. Long-term effect of intravenous immunoglobulin on anti-MuSK antibodypositive myasthenia gravis. Acta Neurol Scand 2007; 116: 406-408.

20. Richman DP, Agius MA. Treatment of autoinmune myasthenia gravis. Neurology 2003; 61: 1652-1661.

21. JANI-ACSADI A, LISAK RP. Myasthenic crisis: Guidelines for prevention and treatment $\mathrm{J}$ Neurol Sci 2007; 261: 127-133.

22. Gajdos P, Chevret S, Clair B, Tranchant C, Chastang C. Clinical trial of plasma Exchange and high-dose intravenous immunoglobulin in myasthenia gravis. Myasthenia gravis clinical study group. Ann Neurol 1997; 41: 789-796.

23. Ronager J, Ravnborg M, Hermansen I, VORSTRUP S. Immunoglobulin treatment versus plasma exchange in patients with chronic moderate to severe myasthenia gravis. Artif Organs 2001; 25: 967-973.

24. ZinMAN L, NG E, BRIL V. IV immunoglobulin in patients with myasthenia gravis. A randomized controlled trial. Neurology 2007; 68: 837-841.

25. RUDNICKI SA. Lambert-Eaton myasthenic syndrome with pure ocular weakness. Neurology 2007; 68: 1863-1864.

26. DHAND UK. Clinical approach to the weak patient in the intensive care unit. Respiratory Care 2006; 51: 1024-1041.

27. Мента S. Neuromuscular disease causing acute respiratory failure. Respiratory Care 2006; 51: 1016-1023.

28. Ito H, Ito H, FujITA K, Kinoshita Y, TAKANAShi Y, LUSAKA $\mathrm{H}$. Phrenic nerve conduction in the early stage of Guillain-Barré syndrome might predict the respiratory failure. Acta Neurol Scand 2007: 116: 255-258.

29. LAWN ND, FLETCher DD, Henderson RD, WOLTER TD, WiJdicks EF. Anticipating mechanical ventilation in Guillain-Barré syndrome. Arch Neurol 2001; 58: 893-898.

30. Murthy JMK, Meena AK, Chowdary GVS, NARYANAN JT. Myasthenic crisis: Clinical features, complications and mortality. Neurology India 2005; 53: 37-40.

31. Agarwal R, Reddy C, Gupta D. Non-invasive ventilation in acute neuromuscular respiratory failure due to myasthenic crisis: case report and review of literature. Emerg Med J 2006; 23: e6. 